\title{
Nonperturbative computation of the time-resolved formation of the profile of autoionizing states as a function of the intensity and duration of ultrashort pulses
}

\author{
Th. Mercouris, ${ }^{*}$ Y. Komninos, ${ }^{\dagger}$ and C. A. Nicolaides ${ }^{\ddagger}$ \\ Theoretical and Physical Chemistry Institute, Hellenic Research Foundation, 48 Vasileos Constantinou Avenue, Athens, GR-11635 Greece
}

(Received 2 December 2010; published 24 January 2011)

\begin{abstract}
By solving the time-dependent Schrödinger equation nonperturbatively by the state-specific expansion approach, we have obtained reliable results showing the dependence of the formation of the excitation profile of the autoionizing state $\mathrm{He}{ }^{\prime} 2 s 2 p^{\prime}{ }^{1} P^{o}$ for Gaussian pulses of intensities in the range $I \approx 10^{10} \mathrm{~W} / \mathrm{cm}^{2}$ to $I \approx 10^{15} \mathrm{~W} / \mathrm{cm}^{2}$, and of duration 100-2 fs. Conclusions are drawn as regards the breakdown of first-order time-dependent perturbation theory.
\end{abstract}

DOI: 10.1103/PhysRevA.83.015403

PACS number(s): $32.80 . \mathrm{Rm}, 31.15 . \mathrm{A}-$

I. Theory and computation of time-resolved quantities concerning the preparation and decay of autoionizing states. Over decades of implementation of quantum mechanics to real atomic and molecular systems, the framework for the theoretical and computational treatment of autoionizing (resonance) states has been time independent. In other words, the concepts and the physically relevant quantities have been and, to an overwhelming extent, are still being determined based on the time-independent Schrödinger equation. For example, Fano's seminal paper of 1961 [1] provided the formalism which can explain in terms of matrix elements on the real energy axis the profile of the photoabsorption cross section from a bound state to a region of a resonance state, the topic of the present paper. Years later, application of the same type of formalism provided the profile for the cross section of a resonance-resonance transition, with a first-principles implementation to the one-photon transition between doubly excited states, $\mathrm{He}^{\prime} 2 s 2 p^{\prime}{ }^{1} P^{o} \rightarrow{ }^{\prime} 2 p 3 p^{\prime 1} D$, for tunable radiation around $3.4 \mathrm{eV}$ [2].

On the other hand, one may argue that resonance formation and autoionization are processes and, as such, are intrinsically time dependent. Therefore, they, and properties connected to them, could in principle be time resolved in real systems, provided the appropriate theoretical and experimental tools exist and are used in a practical manner. As regards theory, it must be able to handle the many-electron problem while solving nonperturbatively and reliably for arbitrary electronic structures and radiation pulses the time-dependent Schrödinger equation (TDSE).

The work reported in this paper serves this frontier discipline. The computational methodology and corresponding analysis utilize the state-specific expansion approach (SSEA), which was initiated about 2 decades ago for the purpose of solving from first principles the many-electron TDSE. In the case of problems involving resonance states, the investigations have been separated into two categories.

\footnotetext{
*thmerc@eie.gr

†ykomn@eie.gr

${ }^{\ddagger}$ caan@eie.gr
}

In the first category, the focus is on the concept and properties of the decaying state, assumed to be established as an unstable polyelectronic wave packet at $t=0$, without memory of the excitation mechanism. Indeed, about 15 years ago $[3,4]$, we demonstrated the possibility of computing from first principles the time-resolved decay of prototypical unstable states of polyelectronic atoms. The computations produced energies, lifetimes, and energy distributions. Most importantly, in view of the well-documented "law" of exponential decay (ED), emphasis was placed on the fundamental issue of determining the magnitudes of the deviation from ED for very long [3,4] and for very short [5] times, using as test cases not simple models and formal manipulations, but real many-electron systems where the operators causing the decay are nonrelativistic or relativistic (Breit-Pauli Hamiltonian) and where the $N$-electron wave functions include the information of electronic structure and of electron correlation.

In the second category, the excitation and the decay of the atomic resonance state are treated as a single coherent process. In 2002, soon after the announcement of the production of single pulses of ultrashort duration in the scale of attoseconds (as) [6], we reported the results and analysis of an $a b$ initio, electronic structure-dependent nonperturbative calculation, which involved the time-resolved effects at the attosecond scale of strong electron-pair correlations [7,8]. Specifically, by including in the Hamiltonian two simultaneous and short excitation pulses, we computed the time-resolved preparation and autoionization of low-lying ${ }^{1} P^{o}$ doubly excited resonance states of $\mathrm{He}$, the corresponding amplitudes of the electron correlation beats, and the time-resolved rearrangement of pairs of electrons into different geometrical locations $[7,8]$.

In this way, the choice of the problems in $[7,8]$ and their quantitative solution in terms of the SSEA demonstrated from the point of view of theory a possible domain of useful applications of spectroscopy at the attosecond scale, namely, the probing of electron correlations in strongly correlated systems and of the interplay between electronic structure and electron dynamics.

A couple of years later, Wickenhauser et al. [9] investigated another aspect of the phenomenology of the time dependence of autoionizing states, using a model of Coster-Kronig fast transitions. Specifically, they looked at the time-differential ionization rate in the vicinity of an autoionizing state, from 
which information about the time-resolved formation of the Fano profile (originating from the discrete-scattering states interference) can be obtained. This idea stimulated us to return to the prototypical helium system and to solve the TDSE so as to obtain from first principles and in a quantitative way the time-dependent preparation of the well-known He $2 s 2 p{ }^{1} P^{o}$ resonance state at about $60.1 \mathrm{eV}$, in terms of the time-resolved formation of its photoabsorption profile which is created by the interaction between an ultrashort pulse and the ground state, $\mathrm{He} 1 s^{2}{ }^{1} S[10]$.

The high level of accuracy of this type of computation eventually led to the recent proposal of using such results for the characterization of ultrashort pulses from free-electron lasers in the regimes of a few femtoseconds (fs) to a few hundreds of attoseconds [11]. In the work of [11], both the analytic and the numerical calculations were done for field strengths below 0.04 a.u.. This is the calculated value above which first-order time-dependent perturbation theory (FOTDPT) [10] starts breaking down for the particular case treated in [11].

II. The problem. In this paper, we report results of the second phase of our recent study of time-resolved features of autoionizing states. This phase concerns the possibility of knowing quantitatively what happens when this problem of time-dependent excitation and autoionization is examined in the regime where FOTDPT breaks down, due either to stronger peak field strengths (with fixed pulse duration) or to longer pulse durations (with fixed peak-field-strength). For this system, the nonperturbative regime which was recognized in our previous publication [11] starts with a peak field strength of 0.04 a.u. $\left(5 \times 10^{13} \mathrm{~W} / \mathrm{cm}^{2}\right)$ and a Gaussian pulse with a full width at half maximum (FWHM), $t_{\mathrm{FWHM}}$, of $10.9 \mathrm{fs}$. The SSEA computations of the present paper were done for $t_{\mathrm{FWHM}}$ of a Gaussian pulse in the range 2-100 fs and for intensities ranging from $I \approx 10^{10} \mathrm{~W} / \mathrm{cm}^{2}$ up to $I \approx 10^{15} \mathrm{~W} / \mathrm{cm}^{2}$.

The excitation scheme whose time dependence we investigated, mainly with respect to the formation of the autoionization profile as a function of field strength and pulse duration, is the following:

$$
\mathrm{He}_{g}\left(1 s^{21} S\right) \underset{\text { one-photon of } 60.17 \mathrm{eV}}{\stackrel{\text { ultrashort Gaussian pulse }}{\longrightarrow}} \Psi\left({ }^{\prime} 2 s 2 p^{\prime}, 1 s \varepsilon p\right),
$$

where $\Psi\left({ }^{\prime} 2 s 2 p^{\prime}, 1 s \varepsilon p\right)$ symbolizes the superposition of the correlated localized wave function, $\Psi_{0}(2 s 2 p)$, with the continuum of scattering states, $U(1 s \varepsilon p)$.

The question is: What are the accurate values of the quantity expressing the differential ionization probability in the region of the autoionizing state, i.e., of

$$
P_{\mathrm{He}}(\varepsilon, t)=\left|\left\langle^{\prime} 1 s \varepsilon p^{\prime} \mid \Psi(t)\right\rangle\right|^{2}, \quad \varepsilon \approx 35.6 \mathrm{eV}
$$

when computed for field strengths and pulse durations which start destroying the regime of FOTDPT for this system? In Eq. (2), $\Psi(t)$ is the solution of the TDSE to all orders, where the Hamiltonian includes the atom-field interaction in the electric dipole approximation for linearly polarized light.
$\mathrm{He}$

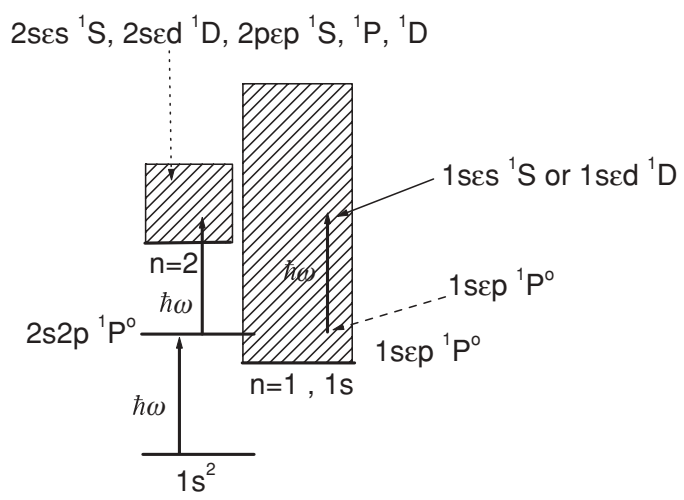

FIG. 1. The excitation scheme of He $1 s^{2}{ }^{1} S$ by an intense short Gaussian pulse with $\omega=60.17 \mathrm{eV}$, implemented in the present SSEA computations. Apart from the one-photon direct transition to the $\mathrm{He}$ $\left(' 2 s 2 p^{\prime}, 1 s \varepsilon p\right){ }^{1} P^{o}$ stationary state, higher order transitions involving the depicted continua have been included in the expansion.

III. Solution of the problem in the framework of the SSEA. The crucial aspect of the implementation of the SSEA is the understanding of the significance of the state-specific wave functions that play the major role in the time evolution of $\Psi(t)$ for a given Hamiltonian. In our problem, the dominant channel transitions that must be included in the calculation beyond the perturbative regime are depicted in the energy spectrum of Fig. 1. The main component of the time evolution of the system, which is present already at the level of firstorder perturbation theory, is the time-dependent population from the He ground state of the stationary-state superposition of He (' $\left.2 s 2 p^{\prime}, 1 s \varepsilon p\right)$, while, at the same time, the unstable, doubly excited ' $2 s 2 p^{\prime}$ state decays into the $1 s \varepsilon p$ continuum via Coulomb coupling.

Specifically, in addition to the $1 s^{2}, 2 s 2 p^{1} P^{o}$ and the $U(1 s \varepsilon p){ }^{1} P^{o}$ states, the state-specific expansion contains the following wave functions:

(i) The scattering channel states above the $n=2$ shell, $2 s \varepsilon s{ }^{1} S, 2 s \varepsilon d^{1} D$, and $2 p \varepsilon p{ }^{1} S,{ }^{1} P,{ }^{1} D$, with energies $E_{2 s \varepsilon \ell}=E_{2 s}^{\left(\mathrm{He}^{+}\right)}+\varepsilon=-0.5$ a.u. $+\varepsilon$ and $E_{2 p \varepsilon p}=$ -0.5 a.u. $+\varepsilon$. These are the dominant scattering channels that are connected, through the electric dipole coupling, with the $2 s 2 p^{\prime}{ }^{1} P^{o}$ localized component, since the magnitudes of the matrix elements $\left\langle{ }^{\prime} 2 s 2 p^{\prime}{ }^{1} P^{o}|z| n s \varepsilon \ell\right.$ or $\left.n p \varepsilon{ }^{\prime} \ell^{\prime}{ }^{1} L\right\rangle$, with $n>2$, are smaller than those of $\left\langle{ }^{\prime} 2 s 2 p^{\prime 1} P^{o}|z| 2 s \varepsilon\right.$ $\ell^{1} L$ or $\left.2 p \varepsilon p{ }^{1} S,{ }^{1} P,{ }^{1} D\right\rangle$.

(ii) The scattering channel states $1 s \varepsilon s^{1} S$ and $1 s \varepsilon d^{1} D$ with energies $E_{1 s \varepsilon \ell}=E_{1 s}^{\left(\mathrm{He}^{+}\right)}+\varepsilon=-2.0$ a.u. $+\varepsilon$, which are dominantly connected, via dipole coupling, to the $1 s \varepsilon p^{1} P^{o}$ states that host the autoionizing ${ }^{\prime} 2 s 2 p^{\prime}{ }^{1} P^{o}$ state.

In this framework, we made an approximation regarding the energy dependence of the continuum-continuum dipole moment matrix elements $\left\langle 1 s \varepsilon_{1} p^{1} P^{o}|z| 1 s \varepsilon_{2} s{ }^{1} S\right.$ or $\left.1 s \varepsilon_{2} d^{1} D\right\rangle$ : They are assumed to be independent of the energies $\varepsilon_{1}$ and $\varepsilon_{2}$ in a narrow region around $\varepsilon_{1 r}=E_{1 s^{2}}^{(\mathrm{He})}+\omega-E_{1 s}^{\left(\mathrm{He}^{+}\right)}$and $\varepsilon_{2 r}=\varepsilon_{1}+\omega$. The values of these matrix elements correspond to the accurate one for $\varepsilon_{1 r}$ and $\varepsilon_{2 r}$, which are connected resonantly through the ultra-short pulse. This approximation 
enhances computational economy without jeopardizing the required accuracy of the overall calculation.

On the other hand, the dipole matrix elements $\left\langle 2 s 2 p^{1} P^{o}|z| 2 s \varepsilon \ell^{1} L\right.$, or $\left.2 p \varepsilon p{ }^{1} S^{1} P,{ }^{1} D\right\rangle$ are indeed calculated, for each energy $\varepsilon$, using the compact, three-term wave function for the $2 s 2 p^{1} P^{o}$ autoionizing state given in [11].

In view of the above construction, the SSEA form of $\Psi(t)$ is

$$
\begin{aligned}
\Psi(t)= & C_{g}(t) \Psi_{g}\left(1 s^{2}{ }^{1} S\right)+C_{\mathrm{res}}(t) \Psi_{\mathrm{res}}\left(2 s 2 p^{1} P^{o}\right) \\
& +\int_{0} d \varepsilon C(\varepsilon, t) \psi\left(1 s \varepsilon p^{1} P^{o}\right)+\int d \varepsilon C_{1}(\varepsilon, t) \psi\left(2 s \varepsilon s^{1} S\right) \\
& +\int d \varepsilon C_{2}(\varepsilon, t) \psi\left(2 s \varepsilon d^{1} D\right)+\int d \varepsilon C_{3}(\varepsilon, t) \psi\left(2 p \varepsilon p^{1} S\right)
\end{aligned}
$$

$$
\begin{aligned}
& +\int d \varepsilon C_{4}(\varepsilon, t) \psi\left(2 p \varepsilon p^{1} P\right)+\int d \varepsilon C_{5}(\varepsilon, t) \psi\left(2 p \varepsilon p^{1} D\right) \\
& +\int d \varepsilon C_{6}(\varepsilon, t) \psi\left(1 s \varepsilon d^{1} D\right) .
\end{aligned}
$$

When this wave function is substituted into the TDSE,

$$
i \frac{\partial \Psi(t)}{\partial t}=\left[H^{A}+V(\omega, t)\right] \Psi(t), \quad V(\omega, t)=z F g(t) \sin (\omega t)
$$

( $F$ is the field strength and $g(t)$ is the Gaussian form), the system of integrodifferential equations that must be solved for the time-dependent coefficients is compact and transparent. The nonperturbative SSEA solution is then used for the
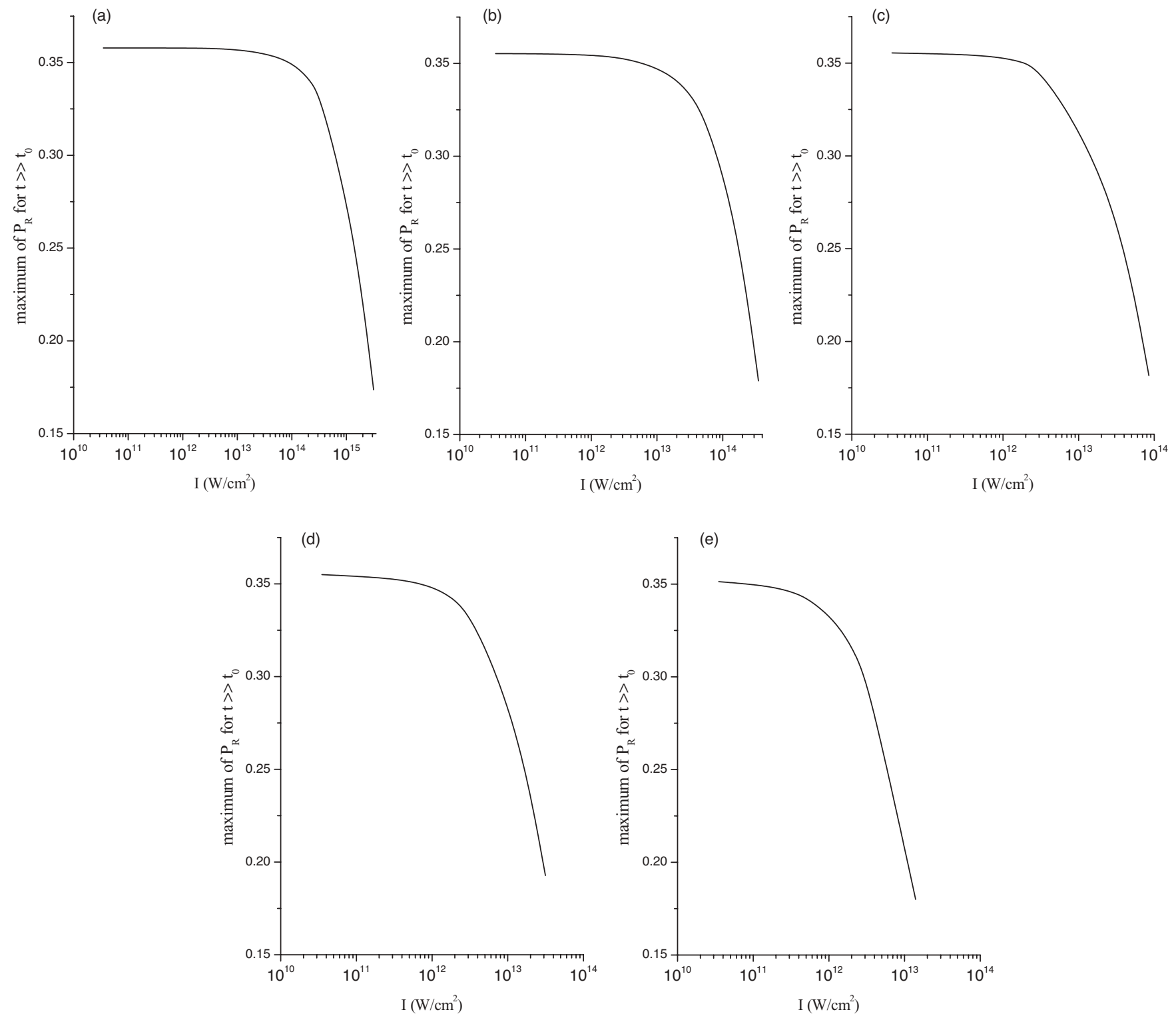

FIG. 2. (a) Quantitative behavior of the main maximum of the reduced differential ionization probability, Eq. (5), for $t_{\mathrm{FWHM}}=2.4 \mathrm{fs}$, as a function of the pulse peak-intensity, $I$. (b) As in panel (a), for $t_{\mathrm{FWHM}}=10.9 \mathrm{fs}$. (c) As in panel (a), for $t_{\mathrm{FWHM}}=25.4 \mathrm{fs}$. (d) As in panel (a), for $t_{\mathrm{FWHM}}=50.8 \mathrm{fs}$. (e) As in panel (a), for $t_{\mathrm{FWHM}}=100.4 \mathrm{fs}$. Comparison of the results of these figures demonstrates quantitatively the distinct effect of the pulse duration on the breakdown of FOTDPT for this problem, in conjunction with the dependence on the pulse intensity. 
computation of Eq. (2), which is $P_{\mathrm{He}}(\varepsilon, t)=|C(\varepsilon, t)|^{2}$, for each set of values of $[F, g(t)]$.

In order to make comparisons with the previous results that are valid in FOTDPT, we recall that in the context of FOTDPT, whose validity rests on the condition that $C_{g}(t) \approx 1$ after the pulse-atom interaction, it is possible to define a "reduced" differential ionization probability as [11]

$$
P_{R} \equiv \frac{P(\varepsilon, t)}{F^{2} t_{\mathrm{FWHM}}^{2}} .
$$

This quantity is independent of $F^{2}$ and of $t_{\mathrm{FWHM}}$ for a Gaussian temporal shape,

$$
g(t)=e^{-2 \ln (2) \frac{\left(t-t_{0}\right)^{2}}{t_{\mathrm{FWHM}}^{2}}},
$$

and for $t \gg t_{0}+\frac{t_{\mathrm{FWHM}}}{\sqrt{2 \ln (2)}}[11]$.

$I V$. Results. The intense fields for this problem, defined as $I>I_{\text {FOTDPT }}$, were determined by comparing the FOTDPT results with those obtained here from the nonperturbative solution of the TDSE. Such a comparison clearly delineates the two regimes as a function of field strength and of pulse duration.

Figures 2(a)-2(e) show the main maximum of Eq. (5) as a function of pulse peak-intensities ranging from $I \approx$ $10^{10} \mathrm{~W} / \mathrm{cm}^{2}$ up to $I \approx 10^{15} \mathrm{~W} / \mathrm{cm}^{2}$, for four different values of the pulse $t_{\mathrm{FWHM}}$, ranging from 2 to $100 \mathrm{fs}$.

At this point, it is appropriate to mention that, in order to obtain convergent results for the aforementioned peak intensities and for large times $\left(t \gg t_{0}+\frac{t_{\mathrm{FWHM}}}{\sqrt{2 \ln (2)}}\right)$, the system of integrodifferential equations of Eq. (3) has to contain up to 90000 equations, which corresponds to a very dense energy mesh for the scattering-state wave functions.

The results show that the value of the main maximum of $P_{R}$ for $I>I_{\text {FOTDPT }}$ decreases quickly.

In addition, the sets of critical values of pulse intensity and duration at which the FOTDPT starts breaking down exhibit the anticipated relationship. Specifically, this breakdown takes place when the critical value of the pulse $t_{\mathrm{FWHM}}$ increases even when the critical value of the peak intensity decreases or the converse. In either case, the FOTDPT condition $C_{g}(t) \approx 1$ loses validity.

V. Conclusion. The significance of the results presented here is twofold. First, they represent a nonperturbative solution of the TDSE for the time-resolved formation of an autoionizing state beyond the limit of FOTDPT. The results are reliable and can serve as reference data for further theoretical or experimental investigations.

Second, the accuracy of such an approach is such that it is possible to draw the following experimentally relevant conclusion when examining the dependence of the profile of $\mathrm{He}$ (or of other prototypical resonance states) on the pulse duration and strength: When the peak intensity is low enough so as to satisfy the requirements of FOTDPT, it is possible to control the temporal characteristics of the ultrashort pulse as described in [11]. Then, it is possible to determine the value of peak intensity, $I$, at which the system enters the nonperturbative regime by monitoring the quantitative behavior of the maximum of $P_{R}$ as a function of $I$.
[1] U. Fano, Phys. Rev. 124, 1866 (1961).

[2] Y. K. Komninos and C. A. Nicolaides, J. Phys. B 30, 811 (1997).

[3] C. A. Nicolaides and T. Mercouris, J. Phys. B 29, 1151 (1996).

[4] T. Mercouris and C. A. Nicolaides, J. Phys. B 30, 811 (1997).

[5] T. Mercouris and C. A. Nicolaides, Phys. Rev. A 65, 012112 (2001).

[6] M. Hentschel et al., Nature (London) 414, 509 (2001).
[7] C. A. Nicolaides, T. Mercouris, and Y. Komninos, J. Phys. B 35, L271 (2002).

[8] T. Mercouris, Y. Komninos, and C. A. Nicolaides, Phys. Rev. A 69, 032502 (2004).

[9] M. Wickenhauser, J. Burgdörfer, F. Krausz, and M. Drescher, Phys. Rev. Lett. 94, 023002 (2005).

[10] T. Mercouris, Y. Komninos, and C. A. Nicolaides, Phys. Rev. A 75, 013407 (2007).

[11] C. A. Nicolaides, T. Mercouris, and Y. Komninos, Phys. Rev. A 80, 055402 (2009). 